\title{
Revisiting the NVSS number count dipole
}

\author{
Prabhakar Tiwari $^{a}$ and Adi Nusser ${ }^{a}$ \\ ${ }^{a}$ Physics Department and the Asher Space Research Institute - Technion, Haifa 32000, Israel \\ E-mail: ptiwari@physics.technion.ac.il, adi@physics.technion.ac.il
}

\begin{abstract}
We present a realistic modeling of the dipole component of the projected sky distribution of NVSS radio galaxies. The modeling relies on mock catalogs generated within the context of $\Lambda \mathrm{CDM}$ cosmology, in the linear regime of structure formation. After removing the contribution from the solar motion, the mocks show that the remaining observed signal is mostly $(70 \%)$ due to structures within $z \lesssim 0.1$. The amplitude of the model signal depends on the bias factor $b$ of the NVSS mock galaxies. For sources with flux density, $S>15 \mathrm{mJy}$, the bias recipe inferred from higher order moments is consistent with the observed dipole signal at $2.12 \sigma$. Flux thresholds above $20 \mathrm{mJy}$ yield a disagreement close to the $3 \sigma$ level. A constant high bias, $b=3$ is needed to mitigate the tension to the $\sim 2.3 \sigma$ level.
\end{abstract}

Keywords: Radio galaxies: high-redshift, Galaxies: active, Cosmology: large scale structure of the Universe, 


\section{Contents}

1 Introduction $\quad 1$

2 Data 2

3 Basics 2

3.1 The dipole component 2

3.1.1 The solar motion contribution to the dipole 3

3.1.2 Contribution from large scale structure $\quad 4$

3.2 The redshift distribution $\quad 4$

$\begin{array}{lll}3.3 & \text { Galaxy biasing } & 4\end{array}$

4 The Mock catalogs $\quad 5$

$\begin{array}{llr}5 & \text { Results } & 7\end{array}$

6 Conclusion and Discussion $r$

7 Acknowledgments 10

\section{Introduction}

The standard cosmological paradigm is based on the "Einstein's Cosmological Principle" [1, 2], i.e. the approach to homogeneity and isotropy on the largest observable scales. Galaxies in the NRAO VLA Sky Survey (NVSS) [3] have been assumed to lie at redshift $z \sim 1$, corresponding to a comoving distance of $\sim 3.3 \mathrm{Gpc}$ for the $\Lambda \mathrm{CDM}$ cosmological background [4]. The galaxy clustering at these distances should contribute very little to sky maps of NVSS galaxy and flux distributions on large angular scales. The observed dipole component of these maps should be almost entirely due to Doppler and aberration effects [5] caused by the local solar motion as measured by the Cosmic Microwave Background Radiation (CMBR) $[6-11]^{1}$. There have been several attempts to measure the dipole in radio catalogs [12-18] and somehow the dipole measured from the NVSS is puzzling with several independent analyses [13-18] yielding values which disagree with the CMBR velocity dipole $[14,17,18]$ at $\sim 3 \sigma$ significance.

The first extraction of the solar motion from the NVSS dipole was done in [13]. The authors [13] reported a velocity which was roughly in the same direction as CMBR but 1.5 to 2 times larger in amplitude. However, nine years later, using the same data, a huge solar motion, $1600 \pm 400 \mathrm{~km}$ $\mathrm{s}^{-1}$, was reported [14] from both the projected number density (per steradian) and brightness dipole measurements. This significant $(>3 \sigma)$ disagreement with the observed solar motion, immediately attracted the attention of the community resulting in further detailed analyses of the NVSS dipole $[15] ;[16]([19]) ;[17,18]$.

The authors of [15] derived a dipole almost six times larger than the CMBR velocity dipole, which was attributed to observational biases. Another analysis by [16] yielded a dipole with an amplitude roughly three times larger than the prediction from the CMBR value. A more recent analysis by [17] found a $2.7-3.2 \sigma$ disagreement between the NVSS number dipole and the CMBR for various flux density cuts. Conclusively, the present status of NVSS dipole is a disagreement with CMBR predicted velocity dipole at $2.7 \sigma$ or more.

However, the contribution of the intrinsic variation of the projected number density as a result of the NVSS galaxy clustering remains to be properly assessed. The intrinsic dipole mainly depends on the following key ingredients:

\footnotetext{
${ }^{1}$ The measured CMBR dipole magnitude is of order $\sim 10^{-3}[6-11]$ and the corresponding speed is found to be $369 \pm$ $0.9 \mathrm{~km} \mathrm{~s}^{-1}$ in the direction, $l=263.99^{\circ} \pm 0.14^{\circ}, b=48.26 \pm 0.03^{\circ}$ in galactic coordinates [10, 11]. In J2000 equatorial coordinates, the direction parameters are $R A=167.9^{\circ}, D E C=-6.93^{\circ}$.
} 
- number density distribution $N(z)$ as a function of redshift. This dictates the fractional contribution of the large scale structure from different redshifts. Available constraints on $N(z)$ strongly indicate that it peaks at redshifts lower than $z \sim 1$.

- relation between the clustering of radio galaxies versus the underlying mass as a function of redshift. This biasing relation can be parametrized in terms of the galaxy, $P_{g}(k)$, and matter power spectrum, $P(k)$, as, $P_{g}(k, z)=b^{2}(z) P(k, z)$. The bias factor, $b$, could be as large as $2-3$ and hence produce a non-negligible boost to the dipole signal, especially if $N(z)$ peaks at redshifts lower than $z \sim 1$.

- constraints from the Local Universe. Our cosmological neighborhood within $100 \mathrm{Mpc}$ is moving with $\sim 300 \mathrm{~km} \mathrm{~s}^{-1}[20]$ and roughly aligned with the solar motion. This motion is caused by even farther structures, producing an alignment between the solar motion and intrinsic dipole.

We aim at a realistic assessment of the NVSS dipole within the context of the $\Lambda$ CDM model taking into account the above ingredients. In Ref. [21] (hereafter NT15), the authors approximate the observed redshift distribution, $N^{\text {obs }}(z)$ from the best available redshift catalogs Combined EIS-NVSS Survey of Radio Sources (CENSORS)[22, 23] and Hercules [24, 25]. They extract the biasing factor $b(z)$ by matching the $\Lambda$ CDM predictions to the NVSS measured angular power spectrum. The redshift dependent biasing and number distribution are also found to be consistent with the observed radio luminosity functions $[23,26,27]$. The results obtained in NT15 are based on high order multipoles $l \geq 4$, where the analysis is fairly insensitive to complications related to the partial sky coverage. In this paper we focus on the dipole, $l=1$, and perform the comparison via $\Lambda$ CDM mock catalogs.

The outline for the paper is as following. In $\S 2$, we briefly describe the NVSS catalog. In $\S 3.1$ we review the basic formalism for describing the dipole from a projected map of galaxies. We provide our treatment for the number density $N(z)$ and galaxy biasing in $\S 3.2 \& \S 3.3$, respectively. $\S 4$ describes the NVSS mock catalogs. We present our results in $\S 5$ and conclude with a general discussion in $\S 6$.

\section{Data}

The full NVSS catalog contains $\sim 1.7$ million sources observed at $1.4 \mathrm{GHz}$ with an integrated flux density $S_{1.4 \mathrm{GHz}}>2.5 \mathrm{mJy}$. The full width at half maximum angular resolution is 45 arcsec and nearly all observations are at uniform sensitivity. Most of the sources (90\%) are unresolved. The catalog covers the sky north of declination $-40^{\circ}$ (J2000), which is almost $82 \%$ of the celestial sphere. However, the effective sky coverage for our study here is $75 \%$ since we impose the following two cuts: 1) we mask out sources at latitudes $|b|<5^{\circ}$ to avoid Galactic contamination and 2) we remove additional 22 sites around bright local extended radio galaxies identified by [13], to avoid overwhelming correlations which may leak to the large scales of interest. These are the only cuts we impose on the data. The authors of [17] have studied the robustness of the measured NVSS dipole with respect to additional cuts. They have demonstrated that masking out latitudes of $<10^{\circ}$, around the Galactic and Super-Galactic planes changes the dipole by less that $10 \%$. The same authors have also assessed the effect of removing sources within 30 arcsec of known nearby galaxies listed in [28-33]. This procedure also resulted in small changes in the NVSS dipole.

As shown by Ref. [13], the NVSS suffers from significant systematic mis-calibration across the sky at flux densities $S \lesssim 15 \mathrm{mJy}$, which affects lower multipoles including the dipole. Therefore, we restrict our analysis to sources brighter than $15 \mathrm{mJy}$ and consistently give the results for $\mathrm{S}>15,20$, 30, 40 and 50 mJy. Furthermore, for completeness and comparison to other authors [13, 16, 17], we give results for $S>10 \mathrm{mJy}$, for one set of mocks with most conservative parameters. We impose a maximum flux density cut $S<1000$ mJy to remove extra bright sources.

\section{Basics}

\subsection{The dipole component}

Let $\mathcal{N}(\hat{\boldsymbol{r}})$ be the projected number density (per steradian) in the line-of-sight direction $\hat{\boldsymbol{r}}$, and $\overline{\mathcal{N}}$ be the mean number density averaged over the sky. Further, let the density contrast $\Delta(\hat{\boldsymbol{r}}) \equiv \mathcal{N}(\hat{\boldsymbol{r}}) / \overline{\mathcal{N}}-1$. 
Given the measured angular positions of galaxies, we employ the HEALPix [34] scheme to obtain $\mathcal{N}(\hat{\boldsymbol{r}})$ in equal area pixels. We expand $\Delta(\hat{\boldsymbol{r}})$ in spherical harmonics, $Y_{l m}(\hat{\boldsymbol{r}})$, as

$$
\Delta(\hat{\boldsymbol{r}})=\sum_{l=1}^{\infty} \sum_{m=-l}^{+l} a_{l m} Y_{l m}(\hat{\boldsymbol{r}}) .
$$

For a full sky coverage, the coefficients, $a_{l m}$ can easily be calculated by inverting equation (3.1), as

$$
a_{l m}=\int_{4 \pi} d \Omega \Delta(\hat{\boldsymbol{r}}) Y_{l m}(\hat{\boldsymbol{r}}) .
$$

The angular power, $C_{l}$, for multipole $l$ is,

$$
C_{l}=\left\langle\left|a_{l m}\right|^{2}\right\rangle .
$$

For a partial sky coverage, we resort to an approximate scheme [35] to compute $C_{l}$,

$$
C_{l}^{\text {obs }}=\frac{\left\langle\left|a_{l m}^{\prime}\right|^{2}\right\rangle}{J_{l m}}-\frac{1}{\overline{\mathcal{N}}}
$$

where $a_{l m}^{\prime}$ is obtained from equation (3.2) but with angular integration over survey region only. Further, $J_{l m}=\int_{\text {survey }}\left|Y_{l m}\right|^{2} \mathrm{~d} \Omega$ is the approximated correction suggested in reference [35]. The term $\frac{1}{\mathcal{N}}$ describes the contribution of the Poissonian shot-noise. The $C_{l}^{\text {obs }}$ is a measure of anisotropy at angular scale $\sim \pi / l$.

\subsubsection{The solar motion contribution to the dipole}

A moving observer sees a brighter sky in the forward direction due to Doppler boosting and aberration effects [5]. The flux density (energy per area per frequency) of sources in NVSS data follows a powerlaw dependence on frequency $\nu, S \propto \nu^{-\alpha}$, with $\alpha \approx 0.75$ [5]. Let $v$ be speed of the observer relative to a source emitting radiation intrinsic frequency $\nu_{\text {rest }}$. To lowest order in $v / c$, the observer sees the radiation at a frequency $\nu_{\mathrm{obs}}=\nu_{\mathrm{rest}} \delta$, where

$$
\delta \approx 1+(v / c) \cos \theta
$$

and $\theta$ is the angle between $v$ and the line of sight to the source. Therefore, the observed flux density is [5],

$$
S_{\text {obs }}=S_{\text {rest }} \delta^{1+\alpha}
$$

at a fixed frequency in observer's frame. The extragalactic radio source population count above some flux density cut, follows a power law $n(>S) \propto S^{-x}$ [5], where $x \approx 1$ [17]. Therefore, the number count above some $S_{\text {obs }}$ will change by a factor $\delta^{(1+\alpha) x}$ due to Doppler effect. In addition, the aberration of light changes the solid angle as,

$$
d \Omega_{\mathrm{obs}}=d \Omega_{\mathrm{rest}} \delta^{-2} .
$$

Using these equations, the dipole in the projected number density due to the local motion, at leading order in $v / c$, is $[5,17]$,

$$
\vec{D}=[2+x(1+\alpha)](\vec{v} / c) .
$$

The dipole term of the power spectrum, $C_{1}$, corresponds to to a dipole amplitude, $D(|\vec{D}|)$ as in equation (3.8), given by [15],

$$
C_{1}=\frac{4 \pi}{9} D^{2}
$$




\subsubsection{Contribution from large scale structure}

In linear theory for gravitational instability, the theoretical angular power spectrum $\tilde{C}_{l}$ is given in terms of the 3D mass density power spectrum $P(k)$ at $z=0$ as [21]

$$
\begin{aligned}
\tilde{C}_{l} & =<\left|\tilde{a}_{l m}\right|^{2}> \\
& =\frac{2}{\pi} \int d k k^{2} P(k) W^{2}(k),
\end{aligned}
$$

where $W(k)=\int_{0}^{\infty} D(z) b(z) p(r) d r j_{l}(k r)$ is the window function in $k$-space and $P(k)$ is the $\Lambda$ CDM mass density power spectrum. Further, $D(z)$ is the linear growth factor normalized to unity at $z=0$ and $p(r) \mathrm{d} r \propto N(z) \mathrm{d} z$ is the probability for finding a galaxy in the range $r-(r+\mathrm{d} r)$ and $r$ is the comoving distance corresponding to redshift $z$.

\subsection{The redshift distribution}

We rely on the results of NT15 for biasing and the redshift distribution for radio galaxies. These authors modeled the redshift distribution of NVSS galaxies on the basis of the observed distribution $N^{\text {obs }}(z)$ obtained from CENSORS and Hercules surveys. The CENSORS catalog was developed to study the evolution of the steep-spectrum radio luminosity function and the survey is presumably complete for flux densities $S>7.2 \mathrm{mJy}$ at $1.4 \mathrm{GHz}$, containing 135 sources. Indeed, the catalog covers only $6 \mathrm{deg}^{2}$ of the ESO Imaging Survey Patch D (EISD). The redshift measurements of the sample are $73 \%$ spectroscopically complete and for the remaining sources the redshift is estimated by $\mathrm{Kz}$ or Iz magnitude-redshift relation. The Hercules redshift survey of 64 sources within $1.2 \mathrm{deg}^{2}$ is complete for $S>2 \mathrm{mJy}$ at $1.4 \mathrm{GHz}$. We combine CENSORS+Hercules data to model NVSS source redshift distribution. We find 133 sources above $10 \mathrm{mJy}$ flux density $S$. For higher flux density cuts we use the same as the number of sources reduces significantly and effectively there is no change in fit. We add that the CENSORS+Hercules combined data sets have been used to study the redshift dependent properties[21-23]. NT15 use the parametric model

$$
N^{\text {model }} \propto z^{a_{1}} \exp \left[-\left(\frac{z}{a_{2}}\right)^{a_{3}}\right],
$$

where the parameters $a_{1}, a_{2}$ and $a_{3}$ have been fixed by fitting the CENSORS+Hercules redshift distribution as well as the NVSS angular power spectrum for $l>4$. The best fit values were found to be $a_{1}=0.74 \pm 0.57, a_{2}=0.71 \pm 0.79$ and $a_{3}=1.06 \pm 0.53$. A histogram of the observed distribution, $N^{\text {obs }}(z)$, is plotted in figure 1 together with the model fit in equation (3.11) for the best fit values of $a_{i}$. Further we have plotted 5 curves representing $N(z)$, for $a_{i}$ randomly drawn from the full probability distribution function computed in NT15.

\subsection{Galaxy biasing}

To model the biasing of radio galaxies, NT15 use an elaborate model based on halo biasing [36] and the relationship between galaxy stellar masses and the prevalence of radio activities [37]. They provide the following convenient parametric fit.

$$
b_{\mathrm{N} T}(z)=b_{1} z^{2}+b_{2} z+b_{3},
$$

where the best fit values of $b_{i}$ are $b_{1}=0.33, b_{2}=0.85$ and $b_{3}=1.6$. Furthermore, we shall employ other choices for the bias factor to fit with NVSS dipole observation. Particularly, we consider constant bias factors $b(z)=2$ and 3 . The thick red line in figure 2 is $b_{\mathrm{N} T}(z)$ for the best fit parameters $b_{i}$, while the thin black lines represent random $b_{i}$ drawn from the full probability function from NT15. Several estimates of the bias factor available in the literature are also plotted in the figure. The values from Allison et al. [38] and Lindsay et al. [39] have a large uncertainty and agree with the bias factors used in this work. However, the bias of faint galaxies $\left(\mathrm{S}_{1.4}>90 \mu \mathrm{Jy}\right)[40]$ is apparently low. The bias measurements in [38-40] correspond to large redshift bins and the redshift for each data points is the median or effective redshift of the bin. The bias of NVSS galaxies obtained by Blake et al. [41] (grey 


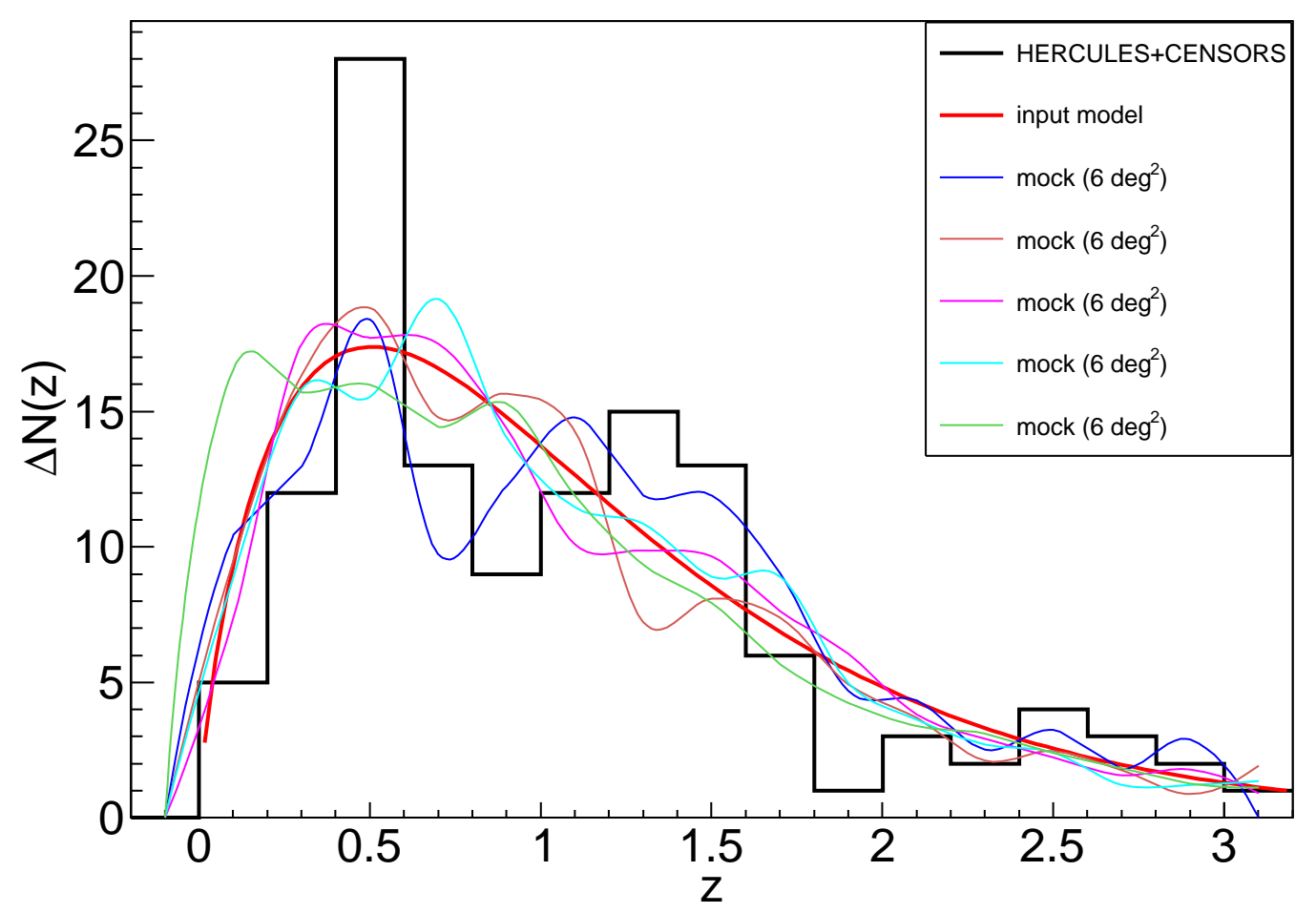

Figure 1. The histogram is the observed number of sources from combined CENSORS+Hercules data sets with flux density $S>10$ mJy and per redshift bin $\Delta z=0.2$. Best fit model (input model) follows equation (3.11) with parameters $a_{1}=0.74, a_{2}=0.71$ and $a_{3}=1.06$. The number density from mock catalogs for same area as observation is also shown. Note that the mocks include the $\Lambda$ CDM density fluctuation, biasing $b(z)$ and the Poisson shot-noise.

band), is assumed to be constant with redshift in their analysis and does not take into account the dipole. Unfortunately, we do not have any radio galaxy bias observation at redshift $z \lesssim 0.1$ where the dipole signal is mostly formed.

\section{The Mock catalogs}

The origin of the intrinsic dipole signature is fluctuations on large scales with negligible coupling to nonlinear evolution on small scales. Hence it suffices for our purposes to construct mock NVSS catalogs based on linear theory for structure formation. The mocks are extracted from a random Gaussian realization of linear density and velocity fields sampled on a $512^{3}$ cubic grid of 17.408 comoving Gpc on the side. The box is equivalent, in volume, to a sphere extending to redshift $z \sim 7$. The grid spacing of $34 \mathrm{Mpc}$ is well below the physical scales relevant for the dipole. The realization of the linear dark matter density, $\delta_{m}$, in the box is generated with the GRAFIC-2 [42] package and the corresponding velocity field is computed using the linear theory relation between velocity and density [43]. We adopt the latest Planck results [4] for the cosmological parameters: Hubble constant $H_{0}=67.8 \mathrm{~km}$ $\mathrm{s}^{-1} \mathrm{Mpc}^{-1}$, total matter density parameter $\Omega_{m}=0.308$, baryonic density parameter $\Omega_{b}=0.0486$, linear clustering amplitude on $8 h^{-1} \mathrm{Mpc}$ scale, $\sigma_{8}=0.815$ and a spectral index $n_{s}=0.9667$. We use the fitting formulae from Ref.[44] for the $\Lambda$ CDM power spectrum. "Observers" are placed at the centers of spheres of $100 \mathrm{Mpc}$ in radius and having with bulk motions of $\sim 300 \mathrm{~km} \mathrm{~s}^{-1}$. This is designed to match the observed bulk flow as measured in [20]. The mean number of the galaxies in a 


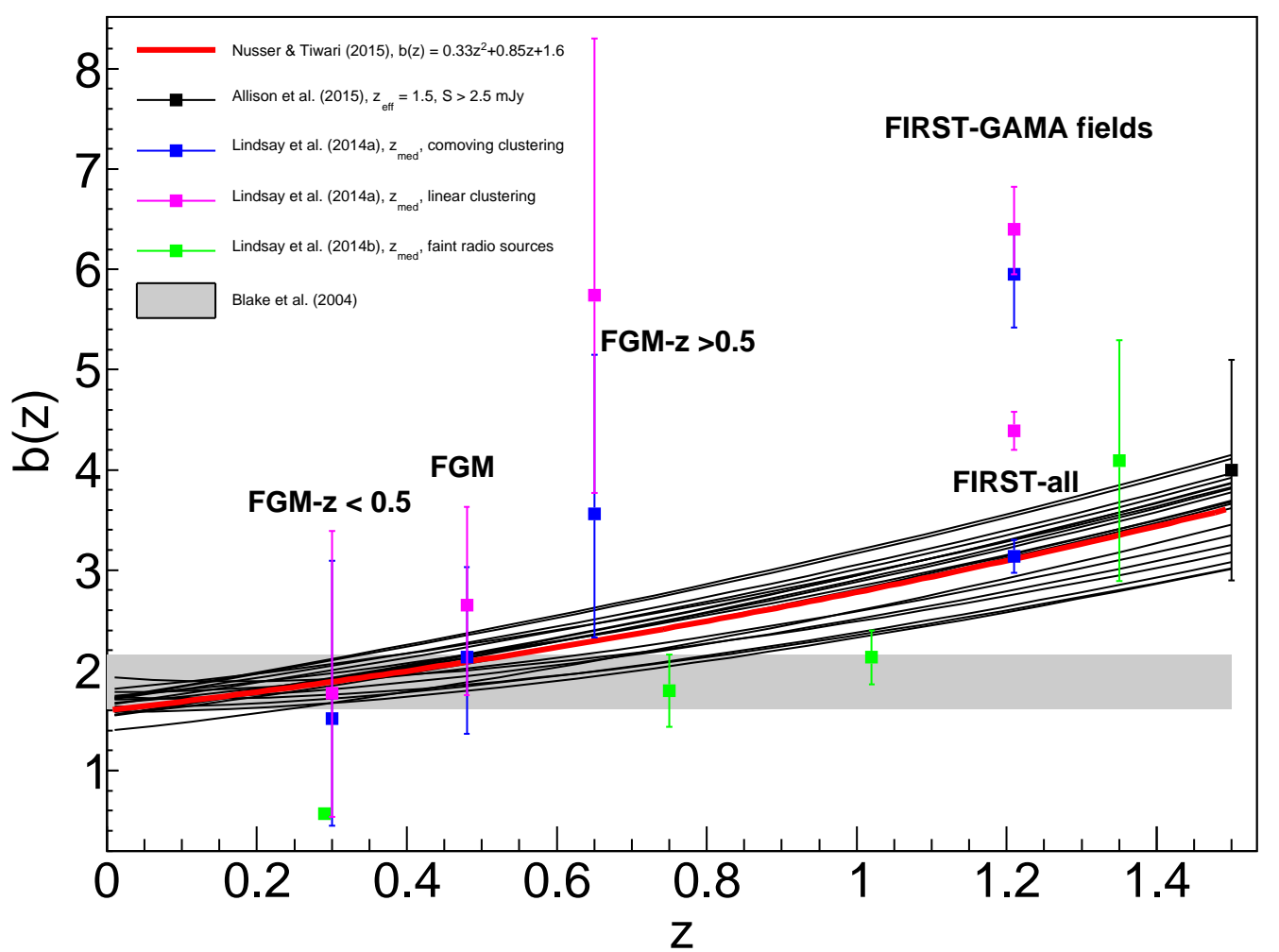

Figure 2. The redshift dependent $b_{\mathrm{N} T}(z)$ in equation (3.12) is plotted as the thick red curve for the best fit parameters. Thin curves correspond to random choices of the parameters appearing in $b_{\mathrm{N} T}(z)$. For comparison, results from the literature are also shown, as indicated in the figure. The abbreviation FGM stands for 'FIRST-GAMA-matched'.

bin at redshift $z_{\text {bin }}, N($ bin $)$, is,

$$
N(\text { bin })=\bar{n}\left(z_{\text {bin }}\right) \times d v \times\left[1+b\left(z_{\text {bin }}\right) D\left(z_{\text {bin }}\right) \delta_{m}\right],
$$

where $\bar{n}\left(z_{\text {bin }}\right)$ is the mean number density at the bin and $d v$ is the bin volume. The number density $\bar{n}\left(z_{\text {bin }}\right)$ is obtained from the redshift distribution form the equation (3.11). The actual number of galaxies in the bin is a random integer, drawn from a Poisson distribution with mean $N($ bin).

In order to include scatter due to uncertainties in the fits of $N(z)$ and for the biasing form $b_{\mathrm{N} T}(z)$ in equation (3.12), we draw random realizations of the parameters $a_{i}$ and $b_{i}$ (see equations (3.11) \& (3.12)) from the full probability distribution for the observed $C_{l}^{\text {obs }}(l>4)$ computed in NT15 (see figures $1 \& 2$ ). $10^{5}$ NVSS mocks are generated, respectively, from these random sets rather than the mean values. The biasing form $b_{\mathrm{N} T}$ is unsuitable for high redshifts but this is insignificant since the contribution to the dipole from large scale structure at $z>1$ practically vanishes (see figure 3 ). For computational purposes we simply take $b(z>1.5)=b(z=1.5)$. When exploring constant bias values, we still generate $10^{5}$ mocks for random shot noise and $a_{i}$. The angular orientation of the observed NVSS sky in relation to the direction of the observed dipole is matched exactly. We rotate the mocked map such as the dipole direction matches with the observed direction and then we mask the Galactic plane and 22 bright local extended sites as identified in [13]. These final mock NVSS galaxy maps are used to calculate $C_{l}$ and are compared with NVSS observed dipole.

It is important to explore the range of redshifts and scales of structures which are mostly responsible for the intrinsic dipole signature. In figure 3 we show the fractional contribution to $\tilde{C}_{1}$ from 
structure up to a certain redshift from the theoretical relation (see equation (3.10)). The dipole is mostly determined by low redshift structure with $60 \%$ of the power already achieved by $z=0.1$.

In figure 4 we show the $k$-space window function, $W(k)$, and the theoretical differential contribution $\tilde{C}_{1}$ to per $\log (k)$ bins. Although the window function peaks around $k \sim 0.001 \mathrm{~h} \mathrm{Mpc}^{-1}$, the overall dipole signal gets most of its contribution from scales $k \sim 0.01 \mathrm{~h} \mathrm{Mpc}^{-1}$.

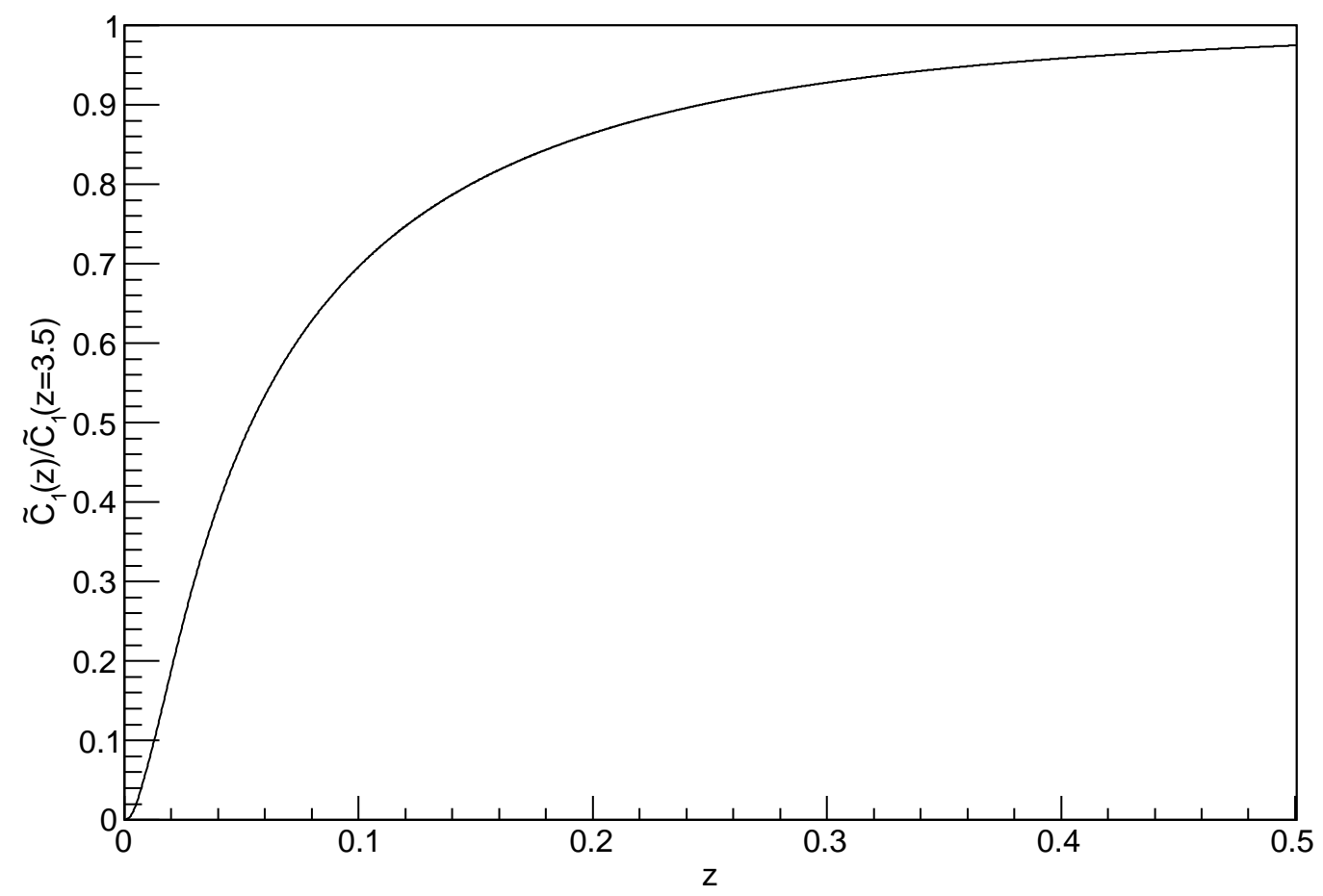

Figure 3. The fractional contribution to $\tilde{C}_{1}$ from structures below redshift $z$.

\section{Results}

The statistical assessment of the ability of the models to match the observed $C_{1}^{\text {obs }}$ is done on the basis of counting the number of mocks with $C_{1}$ exceeding the observed value. We perform this analysis for several lower cuts, $S_{\min }$, on the flux density, $S$, where for each cut we use $10^{5}$ random mocks. The observed $C_{1}^{\text {obs }}$ is calculated after removing the contribution of the solar motion from the NVSS number density maps, according to the relation (3.8).

We first explore consequences of $b(z)=b_{\mathrm{N} T}(z)$ in $(3.12)$ with the parameters which yield a good match to $C_{l}$ for $l>4$ (see NT15). Figure 5 shows the distribution of $C_{1}$ in the $10^{5}$ mocks for $S_{\min }=20 \mathrm{mJy}$. The distribution is well approximated by a $\chi^{2}$ distribution with 3 degrees of freedom corresponding to the average of common variance of three independent Gaussian variables $\left(<\left(\left|a_{11}\right|^{2}+\left|a_{10}\right|^{2}+\left|a_{11}\right|^{2}>\right)\right.$. The theoretical $\chi^{2}$ distribution is also plotted in the figure 5 , as the red curve. The probability for the mocks to yield a $C_{1}$ larger than the observed NVSS dipole $C_{1}^{\text {obs }}$ is $0.2 \%$ for $b_{\mathrm{N} T}$. This corresponds to $\sim 2.89 \sigma$ significance level for a normal distribution of one variable. Values of $C_{1}^{\text {obs }}$ for several flux thresholds, $S_{\text {min }}$, are summarised in table 1 . Note that the NVSS dipole for $S_{\min }=10$ mJy may suffer from systematic biases [13] and we consider it unreliable. We list results with this cut only for the sake of completeness and comparison with other authors. According to the results in table 1 , the $\Lambda \mathrm{CDM}$ mocks with the bias $b_{\mathrm{N} T}$ are reasonably consistent at the $2.12 \sigma$ level with 


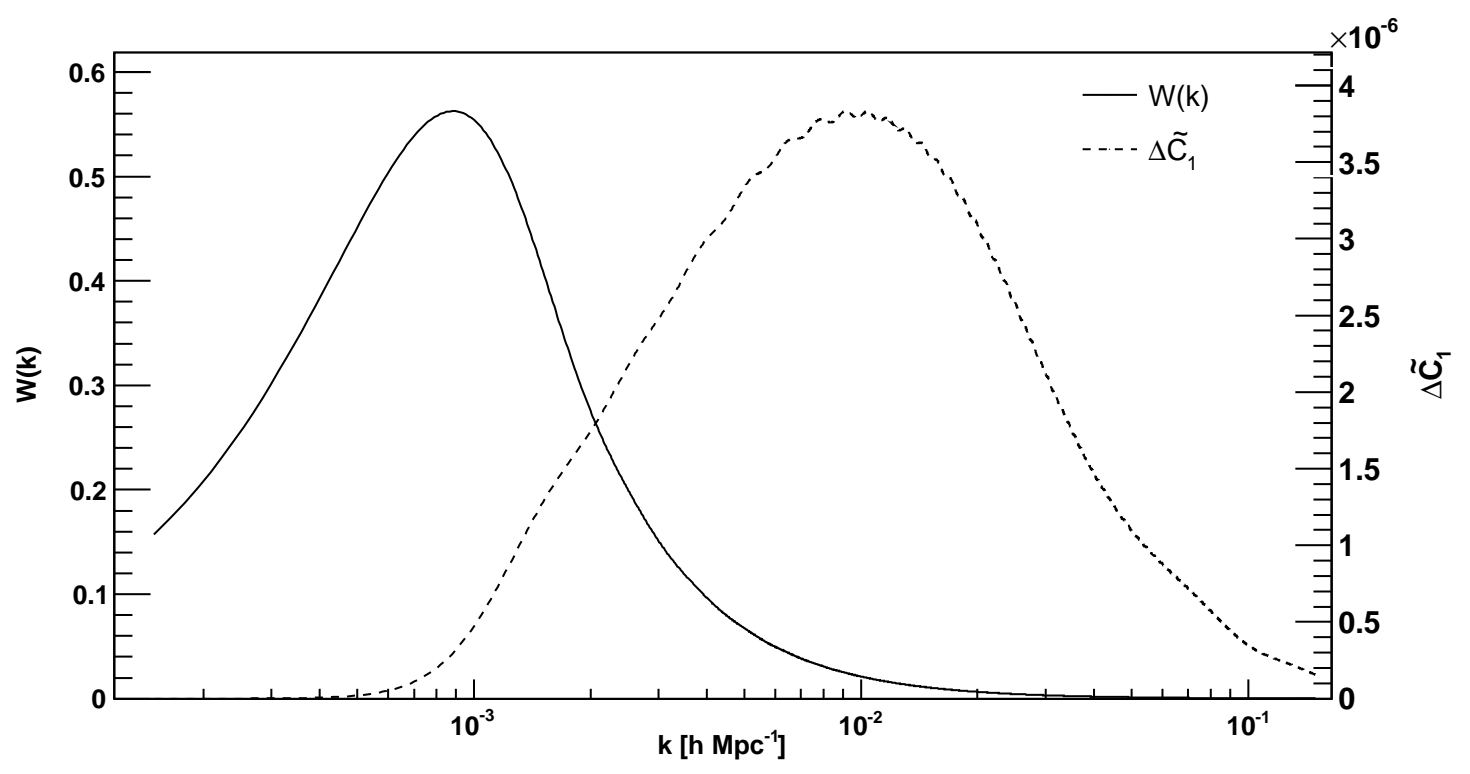

Figure 4. The dipole $k$-space window function, $W(k)$, and the theoretical $\tilde{C}_{1}$ par $\log (k)$ bins. The $\Delta \tilde{C}_{1}$ integral over per $\log (k)$ is normalised to $\Lambda \mathrm{CDM} \tilde{C}_{1}$. The dipole $\left(\tilde{C}_{1}\right)$ is most sensitive to $k \sim 0.01 \mathrm{~h} \mathrm{Mpc}^{-1}$.

the NVSS dipole with $S_{\text {min }}=15 \mathrm{mJy}$. Nonetheless, there is a tension between the mocks and the data at higher fluxes. The biasing template $b_{\mathrm{N} T}$ is inferred from high order multipoles which are sensitive to the galaxy distribution at redshifts $z \gtrsim 0.2$ (see figure 7 in NT15). The dipole signal, however, is largely generated at lower redshifts, $z \lesssim 0.1$ by fluctuations with wave numbers $k \sim 0.01 \mathrm{hMpc}^{-1}$ (cf. figures $3 \& 4)$. Therefore, the choice $b(z)=b_{\mathrm{N} T}$ may not be suitable for the dipole in the case of a redshift and scale dependent (radio) galaxy biasing. Alternatively, we generate mocks for $b(z)=2$ and 3. The results for multipoles $l=1,2,3$ and 4 are shown in table 2 . Both values of constant $b$ are consistent with the dipole for $S_{\min }=15$ and, as with $b_{\mathrm{N} T}$, higher flux thresholds are in tension with the observed dipole for $b=2$. The high value $b=3$ tones down the dipole tension to an acceptable level. However, this value is significantly high compared to most observational estimates [21, 38-41] as seen in figure 2 .

\begin{tabular}{|c|c|c|c|c|c|c|}
\hline$S_{\min }(\mathrm{mJy})$ & $(\mathrm{RA}, \mathrm{Dec})$ & $(l, b)$ & $C_{1}^{\text {obs }}\left(\times 10^{4}\right)$ & shot-noise $\left(\times 10^{5}\right)$ & $\mathrm{p}$-value & $\sigma$ - significance \\
\hline & & & & & & \\
\hline 10 & $\left(135^{\circ}, 23^{\circ}\right)$ & $\left(204^{\circ}, 38^{\circ}\right)$ & 0.82 & & 0.02389 & 1.98 \\
\hline & & & & & & \\
20 & $\left(151^{\circ},-6^{\circ}\right)$ & $\left(246^{\circ}, 38^{\circ}\right)$ & 1.10 & 3.5 & 0.01696 & 2.12 \\
30 & $\left(151^{\circ},-14^{\circ}\right)$ & $\left(253^{\circ}, 32^{\circ}\right)$ & 1.96 & 4.7 & 0.00262 & 2.89 \\
40 & $\left(150^{\circ},-35^{\circ}\right)$ & $\left(263^{\circ}, 36^{\circ}\right)$ & 2.37 & 6.3 & 0.00173 & 2.92 \\
50 & $\left(174^{\circ},-36^{\circ}\right)$ & $\left(286^{\circ}, 16^{\circ}\right)$ & 2.89 & 8.0 & 0.00209 & 2.86 \\
\hline
\end{tabular}

Table 1. The observed $C_{1}^{\text {obs }}$ and shot-noise $\left(\frac{1}{\mathcal{N}}\right)$ along with dipole direction. The probability is the count of mocks with $C_{1}$ exceeding the observed $C_{1}^{\text {obs }}$. The corresponding $\sigma$-significance is also given for the observed probability. The p-value is the probability $\left(C_{1}>C_{1}^{\text {obs }}\right)$. 


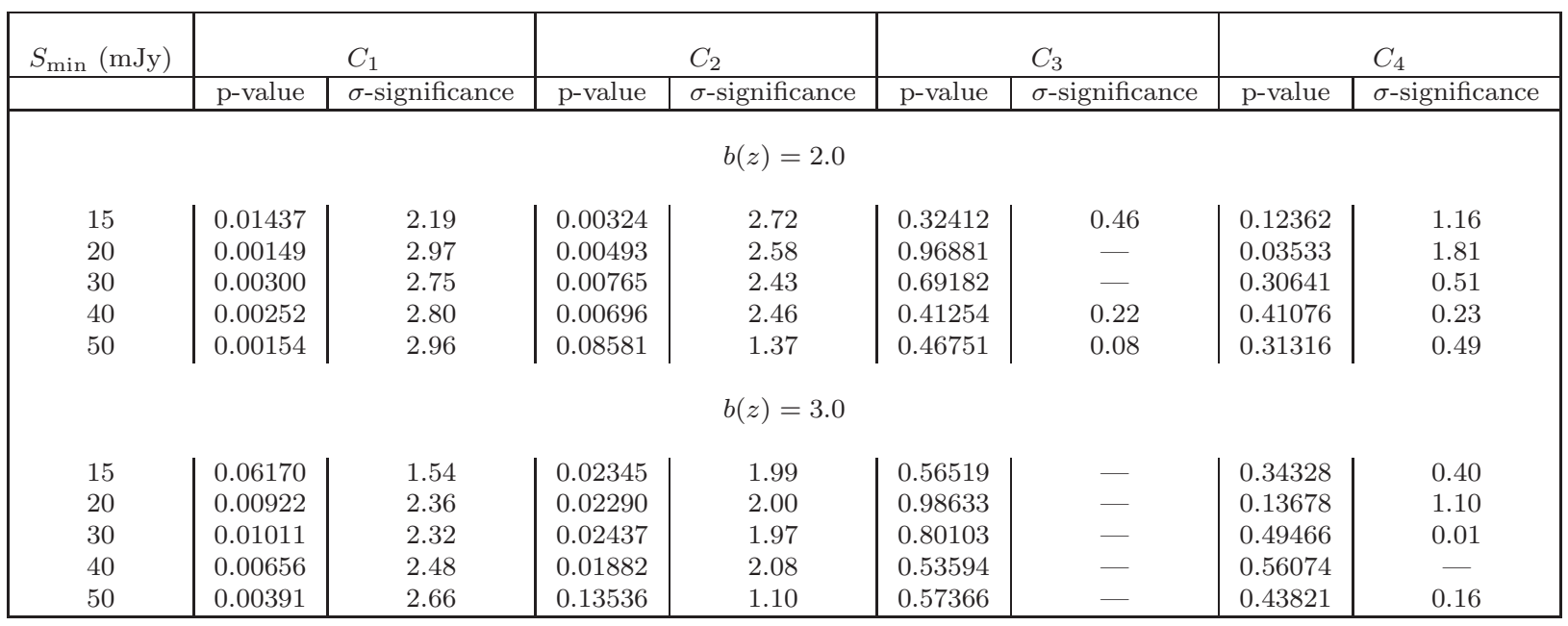

Table 2. The low multipole $C_{1}, C_{2}, C_{3}$ and $C_{4}$ for different biasing templates. The p-value is the probability $\left(C_{l}>C_{l}^{\text {obs }}\right)$. For all p-values $(<0.5)$ we have given the corresponding $\sigma$-significance for a normal distribution of one variable.

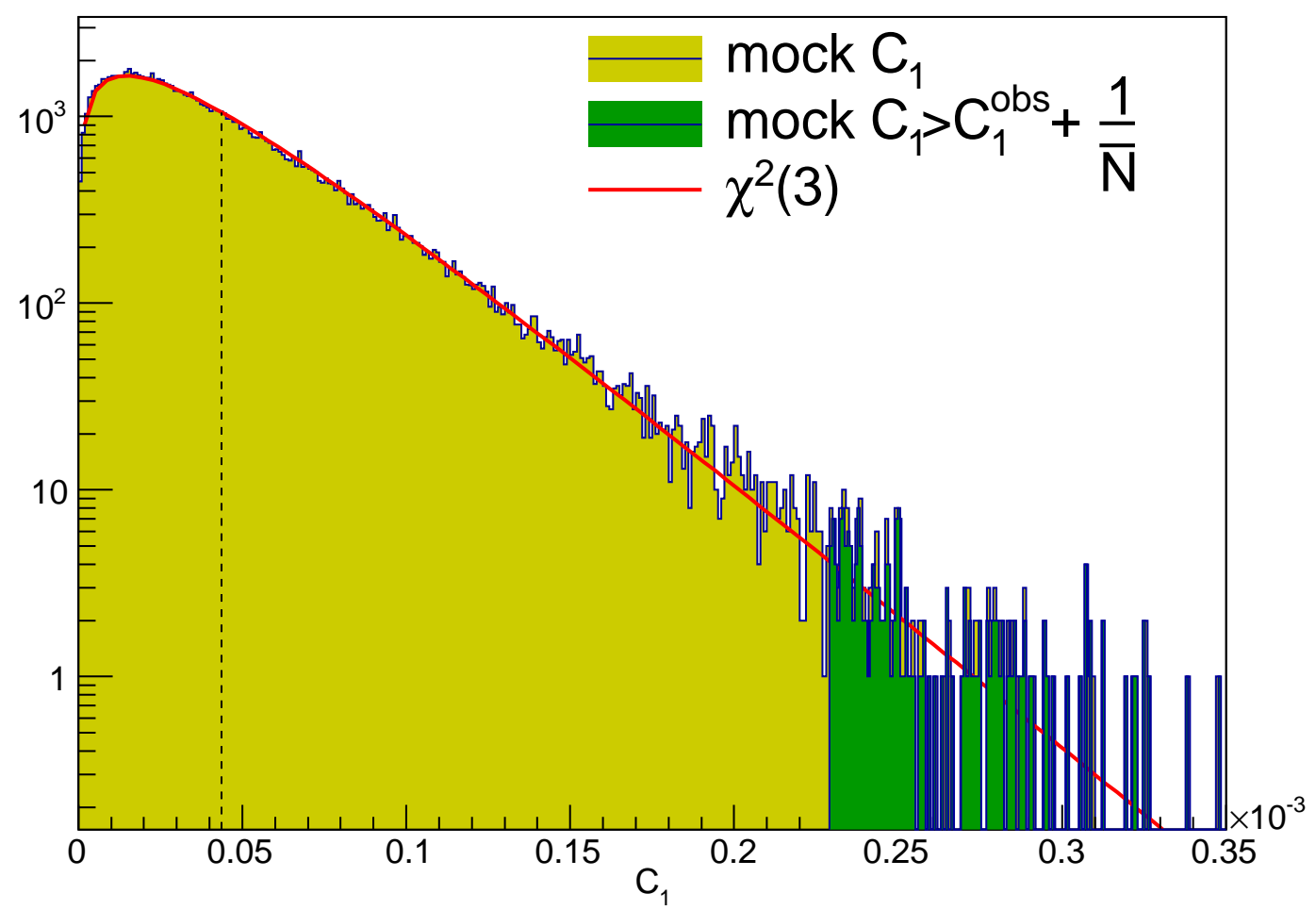

Figure 5. The mocks $C_{1}$ distribution and the observation probability from 1,00,000 samples is shown for the flux density cut $S>20 \mathrm{mJy}$. The dotted line is mean $C_{1}$. Note that the y-axis is in $\log$ scale as the probability for NVSS observed dipole or a dipole with larger amplitude is extremely tiny $(0.2 \%)$ and not even visible in linear scale. A good fit to mocks $C_{1}$ distribution to $\chi^{2}(3)$ probability distribution is also shown. 


\section{Conclusion and Discussion}

We have presented an analysis of the NVSS dipole incorporating, for the first time, the contribution from the large scale inhomogeneities in the galaxy distribution. Mock catalogs mimicking the NVSS catalog are used to model uncertainties in the galaxy redshift distribution and incomplete angular coverage of the data. The bias factor obtained from $l \geq 4$ by NT15 is at odds with the number count dipole measurement at the $\sim 2.9 \sigma$ significance level for the $\Lambda$ CDM cosmology. However, the intrinsic dipole signal is shapes at low $(z \lesssim 0.1)$ redshifts and on large scales $\left(k \sim 0.01 \mathrm{hMpc}^{-1}\right)$; about $70 \%(45 \%)$ of the dipole $(l=4)$ contribution is from redshift $z \lesssim 0.1$. Therefore, it is prudent to consider a large bias factor for radio galaxies at low redshifts as an explanation to the dipole signal. Independent bias estimates at such low redshift are crude, as the redshift of radio galaxies is generally unknown and we do not have much galaxies in low redshift bins. A direct assessment of the redshift dependence of the bias of radio galaxies is done in wide redshift bins due to the small number of radio galaxies with redshift information. A summary of some bias factor determination in the literature is given figure 2 . The radio galaxy bias at low redshift $z \lesssim 0.1$ is loosely constrained and if the bias is as high as $b(z \lesssim 0.1) \approx 3$, it tones down the disagreement between the model and the data to a $\sim 2.3 \sigma$ significance level for $S>20,30,40$ and $50 \mathrm{mJy}$. This bias value agrees, within the large error bars, with the estimate obtained in Ref.[39] (see figure 2). But it is substantially high with respect to other results [38, 40,41], including the value obtained by NT15 for the same NVSS data from higher multipoles $l \geq 4$. We emphasize that the bias extracted in NT15 follows the detailed halo biasing recipe [36] and a relation between galaxy stellar mass and radio activity [37].

The NVSS galaxy sky distribution may be affected by the presence of a large scale mass distribution which generates the observed bulk motion of a sphere of radius $100 h^{-1} \mathrm{Mpc}$ around the observer. The modeling here incorporates the presence of this mass fluctuation by selecting "observers" satisfying the bulk motion constraint. We also rotate the maps so that the dipole signal is aligned with the observation mask as in the real data. As seen in table 3, these two constraints give modest improvement in matching the model to the observations.

There are certain claims in the literature for excess of power on scales of 100s of Mpcs relative to the $\Lambda$ CDM model [45-47]. We will refrain from a detailed assessment of these claims and we simply emphasize the following point. The dipole signal is matched for the $\Lambda \mathrm{CDM}$ for $b=3$. Therefore, any such power excess must be 3 fold higher than the $\Lambda$ CDM. Such large excess at $z \sim 0.1$ will generate a bulk flow within a $100 h^{-1} \mathrm{Mpc}$ which is 3 times the predicted value of the bulk flow in the $\Lambda \mathrm{CDM}$, i.e. a motion of $\sim 800 \mathrm{~km} \mathrm{~s}^{-1}$. Such a value of the bulk flow is at odds with the highest bulk flow estimates in the literature.

There are several non-conventional explanation for the NVSS dipole. For example, the anisotropic mode generated before inflation may re-enter the horizon at late time and produce large scale anisotropy [48-50]. Alternatively, the primordial non-Gaussianities (NG) may affect the structures at large scales. Particularly, significant NG in inflationary cosmology scenario may potentially give an increase in biasing over large scale and produce excess dipole signal [51]. In Ref. [52] the authors argue that NVSS dipole anisotropy can be partially explained with a local over- or under density volume. The authors in Ref. [52] constrain themselves to local structures consistent with CMBR dipole and other observations.

The NVSS dipole remains puzzling within the ambit of radio galaxy biasing. The extra-large power required to explain dipole signal is $\sim 3$ fold in comparison to the $\Lambda \mathrm{CDM}$ and very unlikely. The NVSS excess dipole is most likely due to a residual calibration error across the sky which also affects the high flux cuts. The radio galaxy dipole observation will immensely improve with the Square Kilometer Array.

\section{Acknowledgments}

We thank Ranieri Baldi for discussion and Curtis Saxton for reading the manuscript. This research was supported by the I-CORE Program of the Planning and Budgeting Committee, THE ISRAEL SCIENCE FOUNDATION (grants No. 1829/12 and No. 203/09), the Asher Space Research 


\begin{tabular}{|c|c|c|c|}
\hline$S_{\text {min }}(\mathrm{mJy})$ & 15 & 20 & 30 \\
\hline \multicolumn{3}{|c|}{ considering all constraints } \\
p-value & 0.01696 & 0.00193 & 0.00262 \\
$\sigma$ - significance & 2.12 & 2.88 & 2.79 \\
No velocity constraint \\
p-value & 0.01625 & 0.00175 & 0.00224 \\
$\sigma$ - significance & 2.14 & 2.92 & 2.84 \\
No rotation to map \\
p-value & 0.01280 & 0.00136 & 0.00238 \\
$\sigma$ - significance & 2.23 & 3.00 & 2.82 \\
\hline
\end{tabular}

Table 3. The dependence of $C_{1}$ to different constraints used in mocks. The results are show for flux density cut $S>15,20$ and $30 \mathrm{mJy}$.

Institute, and the Munich Institute for Astro and Particle Physics (MIAPP) of the DFG cluster of excellence Origin and Structure of the Universe. This work is also supported in part at the Technion by a fellowship from the Lady Davis Foundation. We have used CERN ROOT 5.34/21 [53] for generating our plots.

\section{References}

[1] E. A. Milne, World-Structure and the Expansion of the Universe, Zeitschrift für Astrophysik 6 (1933) 1.

[2] E. A. Milne, Relativity, gravitation and world-structure. 1935.

[3] J. J. Condon, W. D. Cotton, E. W. Greisen, Q. F. Yin, R. A. Perley, G. B. Taylor, and J. J. Broderick, The NRAO VLA Sky Survey, AJ 115 (May, 1998) 1693-1716.

[4] Planck Collaboration, R. Adam, P. A. R. Ade, N. Aghanim, Y. Akrami, M. I. R. Alves, M. Arnaud, F. Arroja, J. Aumont, C. Baccigalupi, and et al., Planck 2015 results. I. Overview of products and scientific results, ArXiv e-prints (Feb., 2015) [arXiv:1502.01582].

[5] G. F. R. Ellis and J. E. Baldwin, On the expected anisotropy of radio source counts, MNRAS 206 (Jan., 1984) $377-381$.

[6] E. K. Conklin, Velocity of the Earth with Respect to the Cosmic Background Radiation, Nature 222 (June, 1969) 971-972.

[7] P. S. Henry, Isotropy of the 3 K Background, Nature 231 (June, 1971) 516-518.

[8] B. E. Corey and D. T. Wilkinson, A Measurement of the Cosmic Microwave Background Anisotropy at $19 \mathrm{GHz}$, in Bulletin of the American Astronomical Society, vol. 8 of Bulletin of the American Astronomical Society, p. 351, Mar., 1976.

[9] G. F. Smoot, M. V. Gorenstein, and R. A. Muller, Detection of anisotropy in the cosmic blackbody radiation, Physical Review Letters 39 (Oct., 1977) 898-901.

[10] A. Kogut, C. Lineweaver, G. F. Smoot, C. L. Bennett, A. Banday, et al., Dipole anisotropy in the cobe differential microwave radiometers first-year sky maps, ApJ 419 (1993) 1, [astro-ph/9312056].

[11] G. Hinshaw, J. L. Weiland, R. S. Hill, N. Odegard, C. Larson, et al., Five-Year Wilkinson Microwave Anisotropy Probe Observations: Data Processing, Sky Maps, and Basic Results, ApJS 180 (2009) 225, [arXiv:0803.0732]. 
[12] A. Baleisis, O. Lahav, A. J. Loan, and J. V. Wall, Searching for large-scale structure in deep radio surveys, MNRAS 297 (June, 1998) 545-558, [astro-ph/9709205].

[13] C. Blake and J. Wall, Detection of the velocity dipole in the radio galaxies of the nrao vla sky survey, Nature 416 (2002) 150-152, [astro-ph/0203385].

[14] A. K. Singal, Large Peculiar Motion of the Solar System from the Dipole Anisotropy in Sky Brightness due to Distant Radio Sources, ApJL 742 (Dec., 2011) L23, [arXiv:1110.6260].

[15] C. Gibelyou and D. Huterer, Dipoles in the Sky, MNRAS 427 (2012) 1994-2021, [arXiv:1205.6476].

[16] M. Rubart and D. J. Schwarz, Cosmic radio dipole from nvss and wenss, A 6 A 555 (2013), no. A117 [arXiv: 1301.5559].

[17] P. Tiwari, R. Kothari, A. Naskar, S. Nadkarni-Ghosh, and P. Jain, Dipole anisotropy in sky brightness and source count distribution in radio NVSS data, Astroparticle Physics 61 (Feb., 2015) 1-11, [arXiv: 1307.1947].

[18] P. Tiwari and P. Jain, Dipole anisotropy in integrated linearly polarized flux density in NVSS data, MNRAS 447 (Mar., 2015) 2658-2670, [arXiv:1308.3970].

[19] A. K. Singal, Comment on "Cosmic radio dipole from NVSS and WENSS", Astron.Astrophys. 568 (2014) A63, [arXiv:1408.0679].

[20] A. Nusser and M. Davis, The cosmological bulk flow: consistency with $\Lambda$ CDM and $z \approx 0$ constraints on $\sigma_{8}$ and $\gamma$, Astrophys.J. 736 (2011) 93, [arXiv:1101.1650].

[21] A. Nusser and P. Tiwari, The Clustering of Radio Galaxies: Biasing and Evolution Versus Stellar Mass, ApJ 812 (2015), no. 1 85, [arXiv:1505.06817].

[22] P. N. Best, J. N. Arts, H. J. A. Röttgering, R. Rengelink, M. H. Brookes, and J. Wall, CENSORS: A Combined EIS-NVSS Survey Of Radio Sources - I. Sample definition, radio data and optical identifications, MNRAS 346 (Dec., 2003) 627-683, [astro-ph/0308401].

[23] E. E. Rigby, P. N. Best, M. H. Brookes, J. a. Peacock, J. S. Dunlop, H. J. a. Röttgering, J. V. Wall, and L. Ker, The luminosity-dependent high-redshift turnover in the steep spectrum radio luminosity function: Clear evidence for downsizing in the radio-AGN population, Monthly Notices of the Royal Astronomical Society 416 (2011), no. 3 1900-1915, [arXiv:1104.5020].

[24] I. Waddington, R. A. Windhorst, J. S. Dunlop, D. C. Koo, and J. A. Peacock, The LBDS Hercules sample of mJy radio sources at 1.4GHz - I. Multicolour photometry, MNRAS 317 (Oct., 2000) 801-824, [astro-ph/0006169].

[25] I. Waddington, J. S. Dunlop, J. A. Peacock, and R. A. Windhorst, The LBDS Hercules sample of mJy radio sources at $1.4 \mathrm{GHz}$ - II. Redshift distribution, radio luminosity function, and the high-redshift cut-off, MNRAS 328 (Dec., 2001) 882-896, [astro-ph/0107048].

[26] T. Mauch and E. M. Sadler, Radio sources in the 6dFGS: local luminosity functions at 1.4 GHz for star-forming galaxies and radio-loud AGN, Monthly Notices of the Royal Astronomical Society $\mathbf{3 7 5}$ (Mar., 2007) 931-950, [0612018].

[27] V. Smolčić, E. Schinnerer, G. Zamorani, E. F. Bell, M. Bondi, C. L. Carilli, P. Ciliegi, B. Mobasher, T. Paglione, M. Scodeggio, and N. Scoville, The Dust-Unbiased Cosmic Star-Formation History from the 20 CM VLA-COSMOS Survey, ApJ 690 (Jan., 2009) 610-618, [arXiv:0808.0493].

[28] G. de Vaucouleurs, A. de Vaucouleurs, H. G. Corwin, R. J. Buta, G. Paturel, and P. Fouque, Third Reference Catalogue of Bright Galaxies (RC3). Springer-Verlag, New York, 1991.

[29] H. Corwin, Jr., R. Buta, and G. de Vaucouleurs, Corrections and additions to the third reference catalogue of bright galaxies, AJ 108 (Dec., 1994) 2128-2144.

[30] W. Saunders, W. Sutherland, S. Maddox, O. Keeble, S. Oliver, et al., The PSCz catalogue, MNRAS 317 (2000) 55, [astro-ph/0001117].

[31] T. H. Jarrett, T. Chester, R. Cutri, S. E. Schneider, and J. P. Huchra, The 2MASS Large Galaxy Atlas, AJ 125 (Feb., 2003) 525-554.

[32] J. P. Huchra, L. M. Macri, K. L. Masters, T. H. Jarrett, P. Berlind, et al., The 2mass redshift survey-description and data release, ApJS 199 (Apr., 2012) 26, [arXiv:1108.0669]. 
[33] M. Bilicki, T. H. Jarrett, J. A. Peacock, M. E. Cluver, and L. Steward, Two micron all sky survey photometric redshift catalog: A comprehensive three-dimensional census of the whole sky, The Astrophysical Journal Supplement Series 210 (2014), no. 19.

[34] K. Goŕski, E. Hivon, A. Banday, B. Wandelt, F. Hansen, et al., Healpix - a framework for high resolution discretization, and fast analysis of data distributed on the sphere, ApJ 622 (2005) 759-771, [astro-ph/0409513].

[35] P. J. E. Peebles, The large-scale structure of the universe. Princeton University Press, 1980.

[36] R. K. Sheth, H. J. Mo, and G. Tormen, Ellipsoidal collapse and an improved model for the number and spatial distribution of dark matter haloes, MNRAS 323 (2001), no. 1 1-12, [http://mnras.oxfordjournals .org/content/323/1/1.full.pdf+html].

[37] B. P. Moster, T. Naab, and S. D. M. White, Galactic star formation and accretion histories from matching galaxies to dark matter haloes, MNRAS 428 (2013), no. 4 3121-3138, [http://mnras .oxfordjournals .org/content/428/4/3121.full.pdf+html].

[38] R. Allison, S. N. Lindsay, B. D. Sherwin, F. de Bernardis, J. R. Bond, E. Calabrese, M. J. Devlin, J. Dunkley, P. Gallardo, S. Henderson, A. D. Hincks, R. Hlozek, M. Jarvis, A. Kosowsky, T. Louis, M. Madhavacheril, J. McMahon, K. Moodley, S. Naess, L. Newburgh, M. D. Niemack, L. A. Page, B. Partridge, N. Sehgal, D. N. Spergel, S. T. Staggs, A. van Engelen, and E. J. Wollack, The Atacama Cosmology Telescope: measuring radio galaxy bias through cross-correlation with lensing, MNRAS 451 (July, 2015) 5368-5377, [arXiv:1502.06456].

[39] S. N. Lindsay, M. J. Jarvis, M. G. Santos, M. J. I. Brown, S. M. Croom, S. P. Driver, A. M. Hopkins, J. Liske, J. Loveday, P. Norberg, and A. S. G. Robotham, Galaxy and Mass Assembly: the evolution of bias in the radio source population to $z \sim 1.5$, MNRAS 440 (May, 2014a) 1527-1541, [arXiv:1402.5654].

[40] S. N. Lindsay, M. J. Jarvis, and K. McAlpine, Evolution in the bias of faint radio sources to $z \sim 2.2$, MNRAS 440 (May, 2014b) 2322-2332, [arXiv:1403.0882].

[41] C. Blake, P. G. Ferreira, and J. Borrill, The angular power spectrum of NVSS radio galaxies, MNRAS 351 (July, 2004) 923-934, [astro-ph/0404085].

[42] E. Bertschinger, Multiscale Gaussian random fields for cosmological simulations, Astrophys.J.Suppl. 137 (2001) 1, [astro-ph/0103301].

[43] P. J. E. Peebles, The large-scale structure of the universe. Princeton University Press, 1980.

[44] D. J. Eisenstein and W. Hu, Baryonic Features in the Matter Transfer Function, ApJ 496 (Mar., 1998) 605-614, [astro-ph/9709112].

[45] R. Watkins, H. A. Feldman, and M. J. Hudson, Consistently large cosmic flows on scales of $100 h^{-1} M p c$ : a challenge for the standard $\Lambda$ CDM cosmology, MNRAS 392 (Jan., 2009) 743-756, [arXiv:0809.4041].

[46] E. Macaulay, H. Feldman, P. G. Ferreira, M. J. Hudson, and R. Watkins, A slight excess of large-scale power from moments of the peculiar velocity field, MNRAS 414 (June, 2011) 621-626, [arXiv: 1010.2651].

[47] S. A. Thomas, F. B. Abdalla, and O. Lahav, Excess clustering on large scales in the megaz dr7 photometric redshift survey, Physical Review Letters 106 (Jun, 2011) 241301.

[48] P. K. Aluri and P. Jain, Large Scale Anisotropy due to Pre-Inflationary Phase of Cosmic Evolution, Modern Physics Letters A 27 (2012) 50014, [arXiv:1108.3643].

[49] P. Rath, T. Mudholkar, P. Jain, P. Aluri, and S. Panda, Direction dependence of the power spectrum and its effect on the cosmic microwave background radiation, Journal of Cosmology and Astroparticle Physics 4 (Apr., 2013) 7, [arXiv:1302.2706].

[50] S. Ghosh, Generating Intrinsic Dipole Anisotropy in the Large Scale Structures, Physical Review D. 89 (2014) 063518, [arXiv:1309.6547].

[51] N. Dalal, O. Doré, D. Huterer, and A. Shirokov, Imprints of primordial non-Gaussianities on large-scale structure: Scale-dependent bias and abundance of virialized objects, Physical Review D. 77 (June, 2008) 123514, [arXiv:0710.4560].

[52] M. Rubart, D. Bacon, and D. J. Schwarz, Impact of local structure on the cosmic radio dipole, A\&SA 565 (May, 2014) A111, [arXiv:1402.0376]. 
[53] R. Brun, F. Rademakers, et al., ROOT web page, http://root.cern.ch/, 2001. 